\title{
Condiciones para promover el desarrollo de la competencia de argumentación en el aula de matemáticas*
}

\author{
Conditions to Promote the Development of Argumentation Competence in \\ the Mathematics Classroom
}

Horacio Solar **

Jordi Deulofeu***

\begin{abstract}
Resumen
El foco de este estudio es la gestión de la competencia de argumentación en el aula de matemática. El grupo de investigación Competencias Matemáticas (COMMAT) ha venido realizando investigaciones sobre la formación de profesores para el desarrollo de las competencias matemáticas, entre ellas la argumentación. En esta investigación presentamos, mediante el estudio de casos de clases de matemáticas, las percepciones de los profesores sobre la argumentación en el aula de matemáticas; la existencia de argumentación en las clases observadas; la estructura de la argumentación; el rol del docente para promover el desarrollo de la argumentación; y finalmente, las condiciones para promover la argumentación en la clase de matemáticas, punto que constituye el tema central del artículo. Como conclusión encontramos tres grandes condiciones: tres estrategias comunicativas para promover la argumentación, tareas matemáticas abiertas y una planificación con una gestión especializada de la argumentación.
\end{abstract}

Palabras-clave: Competencias matemáticas. Argumentación. Modelo de Toulmin. Estrategias comunicativas.

\begin{abstract}
The focus of this study is the orchestration of the argumentation competence in the mathematics classroom. The research group Mathematical Competence (COMMAT) has been conducting researches in teacher training for the development of mathematical competences, including argumentation. In this research we present, through case studies in the mathematics classroom, teachers perceptions of argumentation in the mathematics classroom; the existence of argumentation in the classes observed; the structure of the argument; the teacher's role in promoting development of argumentation; and finally, the conditions to promote argumentation in the mathematics classroom, a point which is the focus of this paper. In conclusion, there are three relevant conditions, three communication strategies to promote argumentation, open mathematical tasks, and a class plan for argumentation orchestration.
\end{abstract}

Keywords: Mathematical competences. Argumentation. Toulmin model. Communication strategies.

\footnotetext{
* Una versión preliminar de la investigación se encuentra disponible en CONFERENCIA INTERAMERICANA DE EDUCACIÓN MATEMÁTICA, 14., 2015, Tuxla Guterrez., con el nombre Condiciones para promover la argumentación en el aula de matemáticas.

Doctor en Didáctica de las Matemáticas por la Universidad Autónoma de Barcelona (UAB). Profesor asistente, Pontificia Universidad Católica de Chile, Santiago, Chile. Dirección postal: Av. Vicuña Mackenna, 4860, Macul, CP 7820436, Santiago, Chile.E-mail: hsolar@uc.cl.

**** Doctor en Ciencias de la Educación por la Universidad Autónoma de Barcelona (UAB). Profesor titular, Universidad Autónoma de Barcelona, Bellaterra, Barcelona, Espanã. Dirección postal: Plaza Cívica, s/n, CP 08193 Bellaterra, Barcelona, España. E-mail: jdeulofeu@uab.cat.
} 


\section{Introducción}

Actualmente, el enfoque por competencia es considerado, en la comunidad internacional, como una propuesta educativa que va más allá del aprendizaje de contenidos, y apunta a la formación de ciudadanos constructivos, comprometidos y reflexivos, permitiéndoles identificar y entender el rol que juegan las matemáticas en el mundo (OCDE, 2013). Una de las contribuciones del enfoque por competencias al currículo de matemáticas es dotarle una estructura orientada al desarrollo de procesos matemáticos tales como: argumentar, representar, calcular, modelizar, resolver problemas y comunicar (SOLAR; AZCÁRATE; DEULOFEU, 2012). Además, las competencias matemáticas, al sustentarse en procesos, se caracterizan por ser transversales a los contenidos y desarrollarse a largo plazo de manera cíclica en cada nivel educativo. Así, un enfoque por competencias es coherente con una estructura curricular que destaque los procesos matemáticos.

En el caso de Chile, las actuales Bases Curriculares (MINEDUC, 2013) muestran un salto cualitativo en la importancia que se le da a las competencias, en términos de habilidades, y se propone una organización del currículum que articule habilidades con contenidos. En el área de Matemáticas, se proponen cuatro habilidades concretas a desarrollar: resolver problemas, argumentar y comunicar, modelar, y representar. A partir de los trabajos que hemos desarrollado en la línea de competencias matemáticas, se ha conformado el grupo de investigación Competencias Matemáticas (COMMAT), que contempla tres grandes líneas de investigación: dimensión curricular, desarrollo de competencias en los estudiantes, y formación del profesorado (SOLAR et al., 2014). En relación a la formación de profesores se ha caracterizado una Metodología de Trabajo Docente (MTD) de formación de profesores para el desarrollo de competencias matemáticas (SOLAR et al., 2012), tales como la modelización, la resolución de problemas, la representación, la argumentación y la comunicación. En particular, para este estudio queremos destacar la competencia de argumentar.

En gran parte de las investigaciones se ha utilizado el modelo de Toulmin (1958) para caracterizar el desarrollo de la argumentación en el aula de matemáticas, y si bien la investigación se ha incrementado en este tema, hay pocos estudios que exploren los elementos que promueven la argumentación en el aula. Algunos de estos estudios han evidenciado que las intervenciones del profesor son clave para mejorar los niveles de argumentación en los estudiantes en el aula de matemáticas (CONNER et al., 2014), pero no hay un reporte que aborde otras condiciones para el desarrollo de la argumentación. De este modo, la 
problemática que moviliza la investigación y el objetivo de este artículo consiste en identificar condiciones para promover la argumentación en el aula de matemáticas.

\section{Marco teórico}

En general, los análisis de argumentación en el aula se sustentan en el modelo argumentativo propuesto por Toulmin (1958), que sigue un proceso lineal desde los datos hasta las conclusiones. Esta secuencia consta de seis elementos (GOIZUETA; PLANAS, 2013): Datos, Conclusión, Garantía, Respaldo, Calificador modal, Refutadores (figura 1). Para estudiar la argumentación en el aula de matemáticas, Krummheuer (1995) propone una secuencia de argumentación basada en el modelo argumentativo de Toulmin. Sin embargo, reduce el sistema original a cuatro elementos: datos, garantía, respaldo y conclusión.

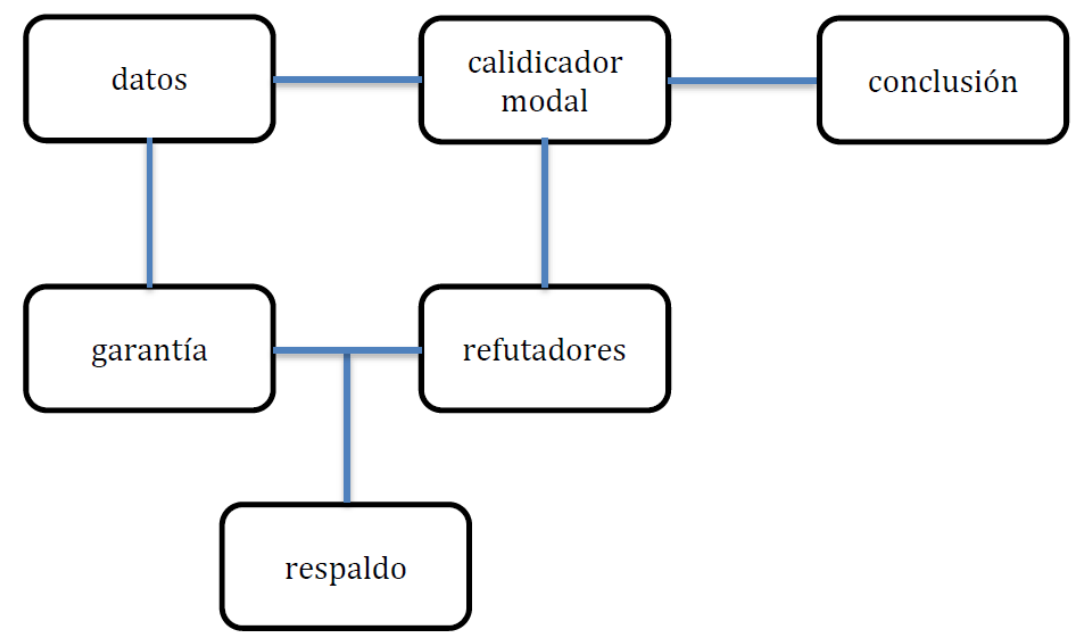

Figura 1- Modelo de Toulmin (1958)

Basándose en el modelo de argumentación de Toulmin (1958), algunos trabajos han estudiado la construcción individual de los argumentos en el aula de matemáticas, mientras que otros investigadores, entre ellos Krummheuer (1995), han destacado la argumentación colectiva como una parte importante del discurso en el aula de matemáticas que incluye cualquier instancia en que el profesor y los estudiantes establecen una sentencia que se puede asociar a la argumentación. Conner et al. (2014) estudian el papel del profesor en la argumentación colectiva, para ello modifican el modelo de Toulmin (1958) para incluir las acciones de los profesores que, si bien no están directamente asociadas a los componentes de la argumentación, contribuyen a su desarrollo. Para nuestra investigación, hemos optado por esta visión de la argumentación colectiva para analizar el aula de matemáticas. 
Para nuestro análisis interpretamos los componentes del modelo argumentativo de Toulmin como procesos matemáticos. En Solar et al. (2012) se muestra un estudio realizado en un curso de $8^{\circ}$ básico sobre qué procesos argumentativos de la estructura de Toulmin (1958) emergen en la implementación de una unidad didáctica de interpretación de graficas funcionales, obteniendo como resultado agregar el proceso de interpretación a la estructura argumentativa.

Si bien la mayoría de los trabajos se enfocan a estudiar la argumentación en el aula de matemáticas, recientemente han aparecido investigaciones que han trasladado el foco a cómo el profesor entiende el desarrollo de la argumentación. Goizueta y Planas (2013) estudian las interpretaciones sobre la argumentación en clase de matemáticas de un grupo de profesores. De los resultados se destaca la dificultad que tienen los profesores para identificar la estructura argumentativa. En esta misma línea, han aparecido otros estudios que utilizan la estructura argumentativa de Toulmin para analizar las respuestas de profesores que se exponen a situaciones hipotéticas de enseñanza y aprendizaje en el aula de matemáticas, en los cuales se han encontrado diferentes tipos de garantías que da el profesor en sus argumentos: de índole didáctico, curricular, pedagógico y personal (NARDI; BIZA; ZACHARIADES, 2012).

Una manera de estudiar los elementos que promueven la argumentación en el aula de matemáticas, es por medio de las estrategias que utiliza el profesor para la gestión de la argumentación. Varios autores han puesto el foco en la importancia de los espacios de comunicación y discusión en el aula de matemáticas (CHAPIN; O'CONNOR; ANDERSON, 2009; SMITH; STEIN, 2011); dichos autores han estudiado los movimientos discursivos del profesor, y el tipo de preguntas para lograr una discusión efectiva en el aula de matemáticas.

En particular, tenemos especial interés en las acciones docentes para promover una comunicación en el aula, que denominamos estrategias comunicativas. Para ello nos hemos basado en Lee (2010), quien señala diversas estrategias que puede utilizar el profesor para incluir a todos los estudiantes en el discurso matemático, entre ellas: hacer preguntas y desarrollar actividades que todos los alumnos consideren que merecen reflexión; fijar y explicitar objetivos que dejen claro que el profesor espera que todo el mundo contribuya; que el profesor se asegure de que todos tengan la oportunidad de aportar algo en un conjunto de temas; hacer explícito a sus alumnos que las respuestas equivocadas revelan errores que el profesor necesita aclarar. Otra estrategia tiene relación con el tipo de preguntas que hace el profesor para guiar el discurso matemático, señalando que una de las formas más importantes que tiene el profesor para orientar el discurso en clase es haciendo preguntas a los alumnos, de 
modo que, mediante el cuestionamiento, el profesor puede detectar dificultades tanto en la comprensión de los conceptos como de los procesos matemáticos (BOERST et al., 2011). El conjunto de estrategias comunicativas señaladas nos parecen clave para analizar la gestión de la argumentación.

\section{Metodología}

La metodología que utilizamos para el logro del objetivo se enmarca en un enfoque cualitativo interpretativo, orientado a describir, interpretar y entender el significado de los fenómenos sociales, intentando darles sentido desde el significado que las propias personas atribuyen a dichos fenómenos (BRYMAN, 2004). Se utilizó un estudio de caso múltiple (YIN, 2014) para entender, desde el discurso y la práctica de los profesores, los procesos de argumentación que emergen en el aula.

Para estudiar el desarrollo de la argumentación consideramos necesario contar con un cuerpo de profesores que la promoviera en el aula, por ello se seleccionó a diez profesores de enseñanza básica de establecimientos educacionales de la ciudad de Concepción para participar de un seminario de formación que ha tenido como propósito estudiar el desarrollo de la argumentación en el aula de matemáticas por medio de estrategias comunicativas.

El seminario se realizó bajo una Metodología de Trabajo Docente (MTD) (SOLAR et al., 2012) cuyo objetivo es que profesores en ejercicio estudien problemáticas en torno a la gestión del aula de matemáticas. La MTD forma parte de una secuencia de cuatro etapas de formación: Estudio de una temática matemática - didáctica; diseño de una secuencia de enseñanza; implementación del diseño; y análisis y reflexión colectiva sobre las distintas experiencias vividas. En todos los pasos se promueve el desarrollo de la reflexión del profesor por medio del análisis de la práctica, utilizando grabaciones de clases. En la primera etapa se analiza la práctica de otros profesores, para pasar, a partir de la segunda etapa, a analizar la propia práctica por medio de grabaciones de sus clases registradas de forma paralela al seminario.

Para analizar las condiciones que promueven la argumentación se han utilizado dos fuentes de datos: por una parte las clases de los profesores participantes del seminario para indagar de qué manera se desarrolla la argumentación en el aula; y por otra parte las sesiones del seminario para analizar las intervenciones de los profesores en relación a sus percepciones sobre el desarrollo de la argumentación en el aula de matemáticas. 
Respecto a la primera fuente de datos, se realizó una observación no participante a ocho clases correspondientes a seis profesoras del seminario (a dos profesoras se le observaron dos clases). Cada una de estas clases fue grabada de manera completa (90 minutos) y se aplicó una pauta de observación, previamente diseñada, en los focos de la investigación: momentos de argumentación y gestión de la argumentación por medio de estrategias comunicación. Como criterio de reducción de datos, entendemos que la discusión en clases y la contraposición de ideas es la base para que exista argumentación (CONNER et al., 2014); en particular, consideramos que en un proceso de argumentación debe existir una estructura de Toulmin que contemple como mínimo la presencia de: dato, garantía, refutación y conclusión. En cuatro de las ocho clases observadas se identificaron momentos argumentativos, que se transcribieron para ser analizados en dos aspectos: identificación de momentos en que hubo argumentación, y caracterización de la estructura de Toulmin.

Respecto a la segunda fuente de datos, se seleccionaron sesiones representativas del seminario en que se analizaron: identificación por parte de los profesores de momentos de argumentación en el análisis de episodios de clase, y caracterización por parte de los profesores de las estructura de Toulmin en los momentos argumentativos identificados.

Por otro lado, para analizar el rol del profesor en el desarrollo de la argumentación, se identificaron las estrategias comunicativas que están presentes en las cuatro clases, para así poder establecer relaciones entre la argumentación y comunicación. Para el proceso de identificación se ha diseñado un instrumento de estrategias comunicativas conformado por acciones docentes que actúan como indicadores. Para caracterizar las estrategias comunicativas nos hemos basado principalmente en la propuesta de Lee (2010). En cambio las acciones docentes han sido establecidas por el equipo de formadores del seminario y compartidas con los profesores, lo que ha tenido como consecuencia una continua revisión de estos indicadores. Una primera versión del instrumento fue validada por un juez experto quien analizó los datos, logrando más del 70\% de acuerdo en el chequeo cruzado, lo que permitió validar los indicadores según los criterios de Miles y Huberman (1994).

En el artículo, nos centraremos en el análisis de dos de los casos del seminario, que son un buen reflejo de los tipos de argumentaciones que se encuentran en el resto de los casos analizados.

\section{Análisis de datos}


El análisis de los resultados se han organizado en 5 temas: significado de la argumentación en la clase de matemáticas; existencia de argumentación en las clases observadas; estructura de la argumentación; rol del docente para promover el desarrollo de la argumentación; y finalmente condiciones para el desarrollo de la argumentación en la clase de matemáticas, punto que responde más directamente a la problemática planteada.

a) Significado de la argumentación

En el aula de matemáticas no es frecuente que encontremos discusiones en las que aparezcan todos los procesos de la argumentación, por lo que acordamos que para que exista argumentación debe haber, por lo menos, cuatro elementos: datos, conclusión, garantía y refutación, siendo este cuarto elemento esencial porque da evidencia de posturas diferentes.

Uno de los primeros resultados que encontramos en la investigación, es la dificultad de los profesores para reconocer la argumentación en el aula de matemáticas. Este resultado se obtuvo en las primeras sesiones del seminario; se presentó a los profesores episodios de aula en video en los cuales los alumnos daban garantías de sus conclusiones, pero en ellos no se presentaban refutaciones que dieran cuenta de puntos de vista diferentes. Por tanto, consideramos que no hay argumentación.

Cuando a los profesores se les pregunta si hay argumentación, la mayoría responde afirmativamente. Este hecho refleja que hay confusión entre explicar y argumentar, ya que la explicación no requiere convencer a otro que tenga una postura contraria. Durante el proceso de formación en el seminario esta percepción en los profesores fue cambiando de manera paulatina, mediante las diferentes tareas que fueron experimentando: análisis de la práctica de otros, de sus pares y de la propia, al ensayar clases que promovieran la argumentación. Todas estas experiencias contribuyeron a mejorar el reconocimiento de la argumentación, lo que se evidencia en la última sesión del seminario en que todos los profesores reconocieron la existencia de argumentación en un determinado episodio de aula.

\section{b) Existencia de argumentación en las clases observadas}

Paralelamente al desarrollo del seminario, se fue a observar a varios profesores a lo que pidió que promovieran la argumentación en clase. No se les entregaron actividades ni indicaciones específicas para esa clase, sino que, a partir de lo que habían percibido hasta ese momento en los seminarios tenían que preparar una clase para promover la argumentación. Se observaron ocho clases y se seleccionaron en base a la existencia de argumentación, es decir, con episodios en los que se identifiquen los cuatro elementos: datos, conclusión, garantía y refutación; de las ocho clases, cuatro cumplieron íntegramente esta condición, mientras que las otras cuatro cumplieron sólo tres: dato, garantía y conclusión, con ausencia de refutación. 
En particular, las primeras cinco clases que se grabaron fueron poco no argumentativas (solo se seleccionó una de ellas), mientras que las tres últimas clases fueron seleccionadas como argumentativas. Esto nos lleva a constatar que: en la medida que los profesores van profundizando en el estudio de la argumentación en el seminario, sus clases son cada vez más argumentativas.

El desarrollo de la argumentación la ilustraremos en un curso de $7^{\circ}$ básico (12-13 años) de un establecimiento educacional subvencionado de la ciudad de Concepción en Chile. La profesora del curso, Matilde, ha trabajado con el equipo de formadores en proyectos anteriores y antes del seminario ya tenía ciertos conocimientos sobre el desarrollo de la argumentación en el aula. La clase observada a dicha profesora tenía como intención promover la argumentación, pero sin contar con un diseño de especial para ello.

Matilde presenta el siguiente problema relacionado con los números enteros: $U n$ número entero y su inverso distan en la recta 12 unidades ¿qué números son?. Matilde espera que surja la solución -6 y 6, y para ello dibuja una recta numérica; ella escucha que la respuesta genérica de los estudiantes corresponde a los números -12 y 12. La profesora en vez de tratar directamente el error, les hace leer nuevamente el problema y pregunta por el significado del término distan; varias de las respuestas de los alumnos le dan como significado la distancia entre 0 y un número, en vez de la distancia entre dos números cualquiera; ello puede explicar los valores dados de 12 y -12 que están a 12 unidades de distancia del 0 cada uno. Matilde, por medio de varias acciones docentes y de la utilización de la recta numérica, sigue trabajando con los estudiantes el significado de distan, para contrastar la idea instalada de que la distancia se cuenta desde 0 hasta el número; entonces, emerge en uno de los estudiantes que el significado de distan se asocia a la distancia entre dos números, y si se eligen -12 y 12 , se debe contar la distancia entre ambos números, que no es 12 sino 24.

Matilde selecciona algunas de las respuestas para ponerlas en común para generar conflicto con la idea instalada de medir la distancia entre 0 y un número, y no pone en común sólo la respuesta de Martín quien señala que la distancia es entre -12 y 12 en vez de 0 y 12 , sino que incentiva a que el resto del curso opine sobre su idea. Al obtener solo 3 respuestas, dirige una nueva pregunta a David respecto a que punto de referencia se mide la distancia, y este sigue sosteniendo que desde el 0, al igual que Roberto y Mario que apoyan a David. A continuación Matilde le da la palabra a Francisco quien es el primero en explicar por qué se mide la distancia desde -12 a 12 , y este refuta la idea instalada de 0 a 12 . Matilde, en lugar de validar la intervención de Francisco, le invita a seguir describiendo su idea para que el resto del curso la escuche; sigue habiendo alumnos, como Isabel, que continúan con la idea de que 
la distancia es entre 0 y 12 , por lo que la profesora pide más opiniones y ante el silencio hace leer nuevamente el enunciado para dilucidar el significado de distan. A partir de su nueva lectura varios alumnos, entre ellos Roberto, señalan que los números tienen que distar 12 unidades, y no del 12 al otro, lo cual contribuye a comprender el enunciado que lo que busca es un número y su inverso cuya distancia es 12 unidades entre ellos. Esta aclaración permite que emerja la idea de Juan, quien dice que debe ser del 0 al 6 para que diste 12 unidades. En este momento, Matilde le incentiva a seguir repitiendo su idea, y Juan señala como respuesta que los números son -6 y 6 . Matilde no valida la respuesta sino que sigue promoviendo que otros estudiantes opinen sobre este resultado, entre ellos Roberto quien no está de acuerdo con los números señalados y sigue sosteniendo que la respuesta es -12 y 12.

Otro caso interesante para mostrar el desarrollo del conflicto en la argumentación, se presenta en una clase de $\left.6\right|^{\circ}$ básico de Geometría que tiene por objetivo: Identificar rectas paralelas, perpendiculares y secantes en cuadriláteros. Para el desarrollo de la argumentación, la profesora Mónica realiza, en una primera instancia, un momento de discusión para extraer conocimientos previos y, más tarde, una actividad basada en una guía de trabajo individual, en la cual los alumnos deben asociar distintas figuras según las similitudes que poseen. Una vez que Mónica se ha paseado por la sala para ver las diferentes respuestas de los alumnos, proyecta en la pizarra la guía que repartió entre los alumnos y hace pasar a Marcelo y a Fernanda a la pizarra para que marquen que figuras tienen las mismas características. En una de las respuestas no coinciden: Fernanda no marcó un cuadrado como similar al tratarse de un cuadrado cuya base estaba en diagonal a la base de papel, a diferencia de Marcelo que sí lo marcó como similar a otro cuadrado de base horizontal a la hoja del papel. La profesora gestiona que se discutan los dos puntos de vista y Fernanda se convence que las dos figuras tienen las mismas características. La manera como se desarrolla la discusión tienen varios puntos interesantes para el análisis: la profesora Mónica recoge la idea de Marcelo de girar la hoja como explicación para determinar que las dos figuras tienen las mismas características; ante ello, los alumnos se encuentran divididos en sus respuestas y algunos tienen dificultades para inclinarse por una de las dos posturas. En este momento, Mónica interviene para que se identifiquen las dos posturas de manera explícita y promueve que el resto del curso se posicione frente a las dos posturas; entre sus acciones propone que Fernanda gire su guía para verificar la idea de Marcelo, y lo contraste con sus pares. Luego de un tiempo pregunta a Fernanda si cambio su opinión; si bien Fernanda hace una explicación del movimiento, Mónica insiste en la pregunta hasta que Fernanda reconoce su cambio de opinión. 


\section{c) Estructura de la argumentación}

Los dos casos expuestos se analizaron en base a la estructura de Toulmin. La estructura de ambos incluye los cuatro elementos de la argumentación. El caso de la clase de Matilde corresponde a esta estructura: para generar un conflicto la profesora pregunta cuál será la distancia entre -12 y 12 , y el desarrollo de dicha pregunta genera una estructura argumentativa que mostraremos por medio del esquema de Toulmin. Para comprender como actúa este esquema se transcribe el episodio.

1 Matilde: Ya, ¿y qué distancia habría del -12, Daniel, al +12 ?

2 Alumnos: 24.

3 A: No, 12 señorita.

4 Roberto: $O$ sea no, sería 12.

5 Matilde: Tenemos...

6 Roberto: Porque del 0 se empieza a contar de nuevo.

7 A: Sí.

8A: No.

9 Matilde: Ya, si de aquí hasta aquí tenemos una distancia de 12, ¿he llegado al inverso de 12? [Señala en la pizarra la distancia que hay de -12 hasta 0]

10 Alumnos: No.

11 Matilde: Miren, voy de -12 a su inverso, avanzo, ¿cuánto llevo hasta aquí? [Marcando el 0 en la recta numérica]

12 Alumnos: 12.

13 Matilde: Si sigo...

14 Javier: Es como multiplicarlo por 2 y sería 24.

15 Arturo: 24.

16 Matilde: ¿Cuánto avancé para llegar al inverso?

17 Alumnos: 24.

18 Matilde: ¿Cuál es la distancia de -12 hasta 12?

19 Alumnos: 24.

20 Matilde: Distan 24 unidades entre estos dos valores.

21 Alumnos: Sería del -6 al 6 entonces.

El esquema de Toulmin que corresponde a una argumentación colectiva (figura 2), comienza con el dato, en este caso la pregunta de Matilde. Luego vienen dos respuestas que actúan como conclusiones diferentes 24 y 12; para seguir el proceso argumentativo tomaremos como conclusión falsa el 12. Siguiendo la estrategia de análisis de Conner et al. (2014), en los cuadros se han puesto las intervenciones tanto de Matilde como de los alumnos que son parte de la estructura argumentativa, en cambio en los globos se han puesto las intervenciones de Matilde que contribuye por medio de preguntas a que aparezca la refutación a la falsa conclusión y luego a la conclusión verdadera. 


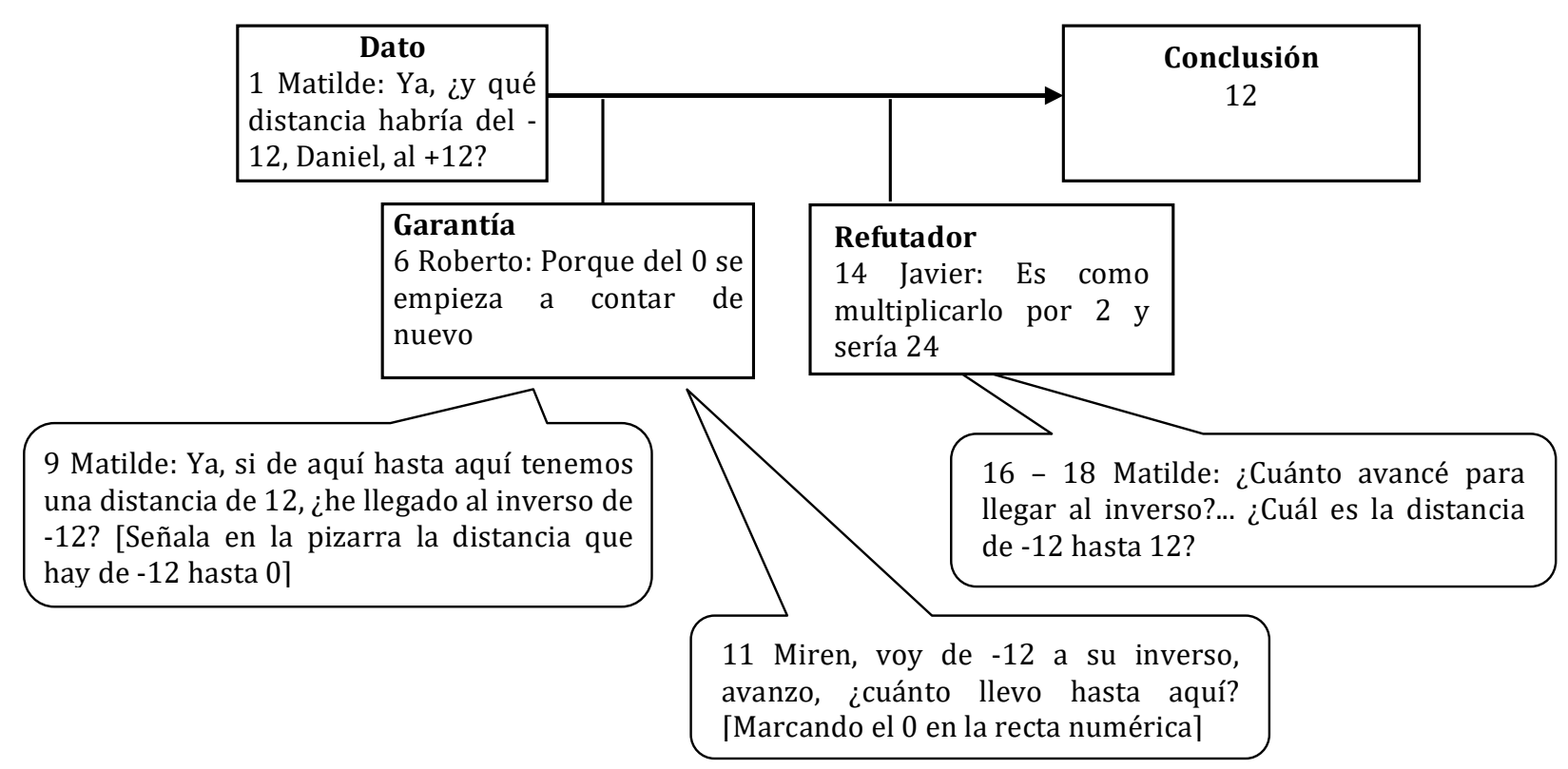

Figura 2- Mapa de la argumentación colectiva según el modelo de Toulmin

Aunque en la clase de Matilde aparecen diferentes momentos argumentativos, hemos escogido este como base de nuestro análisis porque la conclusión original es falsa y emerge la refutación por medio de las preguntas de Matilde. Si bien es la profesora quien comienza con el proceso de argumentación por medio del dato, son los propios alumnos los que van desarrollando la argumentación. Las dos preguntas que hace Matilde promueven que Javier refute la garantía de Roberto; en ese sentido, se aprecia la importancia que tienen las intervenciones de la profesora para gestionar el error por medio de la indagación en el curso, en vez de adoptar una posición que consiste en validar las respuestas de los alumnos.

Si bien el caso descrito da cuenta de un adecuado momento de argumentación, este carece de lo que es el respaldo, es decir de un conocimiento que generalmente corresponde a una definición, propiedad o teorema que permita asegurar la garantía. En los distintos casos analizados, la argumentación se comporta de la misma manera, es decir, se obtienen estructuras de argumentación con cuatro elementos: datos, conclusión, garantía y refutador, pero no emerge por parte de los estudiantes el respaldo a estas argumentaciones, es decir de afirmaciones que sean válidas para todos y que sostengan la argumentación.

En el segundo caso, la clase de la profesora Mónica, se presentó la oportunidad que emergiera el soporte de la garantía. Luego que Sofía se convence de que los dos cuadriláteros corresponden a un cuadrado, Bastián no está de acuerdo y da a entender que cuando gira una de las figuras la otra que era un cuadrado deja de ser un cuadrado. Mónica hace que Josefa y 
Daniel pasen a la pizarra para que discutan esta idea con Bastián y con el resto del curso. En el episodio se aprecia el desarrollo de la discusión.

1 Daniel: Yo pienso que si ese cuadrado...si ese lo damos vuelta va a dar lo mismo que ese. Si lo seguimos dando vuelta, seguimos formando el cuadrado

2 Mónica: $Y a$

3 Bastián: No, pero si se...si se dobla la guía...el otro igual gira

4 Mónica: El otro...el otro...

5 Bastián: Si no tendríamos que tapar este y ver ese como un cuadrado, y (no se escucha bien)

6 Mónica: Ya, a ver Bastián

7 Bastián: Porque si lo dejo así, si lo dejo así (no se escucha)

8 Mónica: Ya, a ver ¿Escuchamos? Bastián dice...el que está al lado izquierdo, cuando lo gira él lo ve como un cuadrado, pero el que era cuadrado lo deja de ver como cuadrado.

Entonces, si tapa el que era cuadrado, dice que ese es un cuadrado

9 Blanca: No tía, está loco

10 Mónica: Pero Blanca, espera. Él dice que es un cuadrado. Ahora, Daniel y la...

11 Bastián: Pero si...

12 Mónica: Pero sigue siendo un cuadrado según tú. Ya, siguen siendo diferentes, pero si lo giro es cuadrado, ¿si o no?

13 Bastián: $\mathrm{Si}$

14 Mónica: Ya, ¿podemos escuchar, Romina, podemos escuchar a Daniel y la Josefa? Porque ellos van a explicar qué es lo que piensan

15 Josefa: Que si uno da vuelta la guía, emm...

16 Daniel: Va a quedar como cuadrado

17 Josefa: Si [Dibuja las figuras en la pizarra, la posición inicial y cómo quedaría al girarla]

18 Daniel: Si la seguimos dando vuelta va a quedar igual como estaba esa

19 Mónica: Pero explíquenles a ellos po chiquillos.

20 Blanca: Pero no...

21 Mónica: Pero Blanca escucha, porque hay que escuchar

22 Daniel: Que si este lo damos vuelta forma como un cuadrado, si lo seguimos dando vuelta igual vamos a tener un cuadrado

23 Josefa: Aunque se gire la figura va a seguir siendo un cuadrado

24 Mónica: Ya, eh... Blanca, pero espera un poco. Espera un poco porque o sino no vamos a...nos vamos a llegar a un acuerdo. La pregunta que les voy a hacer, si yo tengo una figura y la empiezo a girar... ¿pueden tomar atención por fa?...y la empiezo a girar, ¿esa figura va a ser otra figura?

25 Alumnos: No

26 Mónica: ¿O va a seguir siendo la misma figura?

27 Alumnos: Va a seguir siendo la misma.

El esquema de Toulmin que corresponde a una argumentación colectiva (figura 3), se sostiene en un dato que es implícito en la discusión: las dos figuras, ¿son un cuadrado?, y que da pie a la argumentación. Siguiendo a Conner et al. (2014), hemos puesto el dato en un cuadro con una línea entrecortada para diferenciarlo del resto de procesos argumentativos. La primera conclusión emerge de manera implícita de la garantía de Bastían: el que está al lado izquierdo, cuando lo gira él lo ve como un cuadrado, pero el que era cuadrado lo deja de ver como cuadrado. Daniel refuta la idea de Bastían diciendo que el cuadrado al girarlo no deja de ser cuadrado y Josefa se sostiene de esa idea para llegar a esa conclusión. 


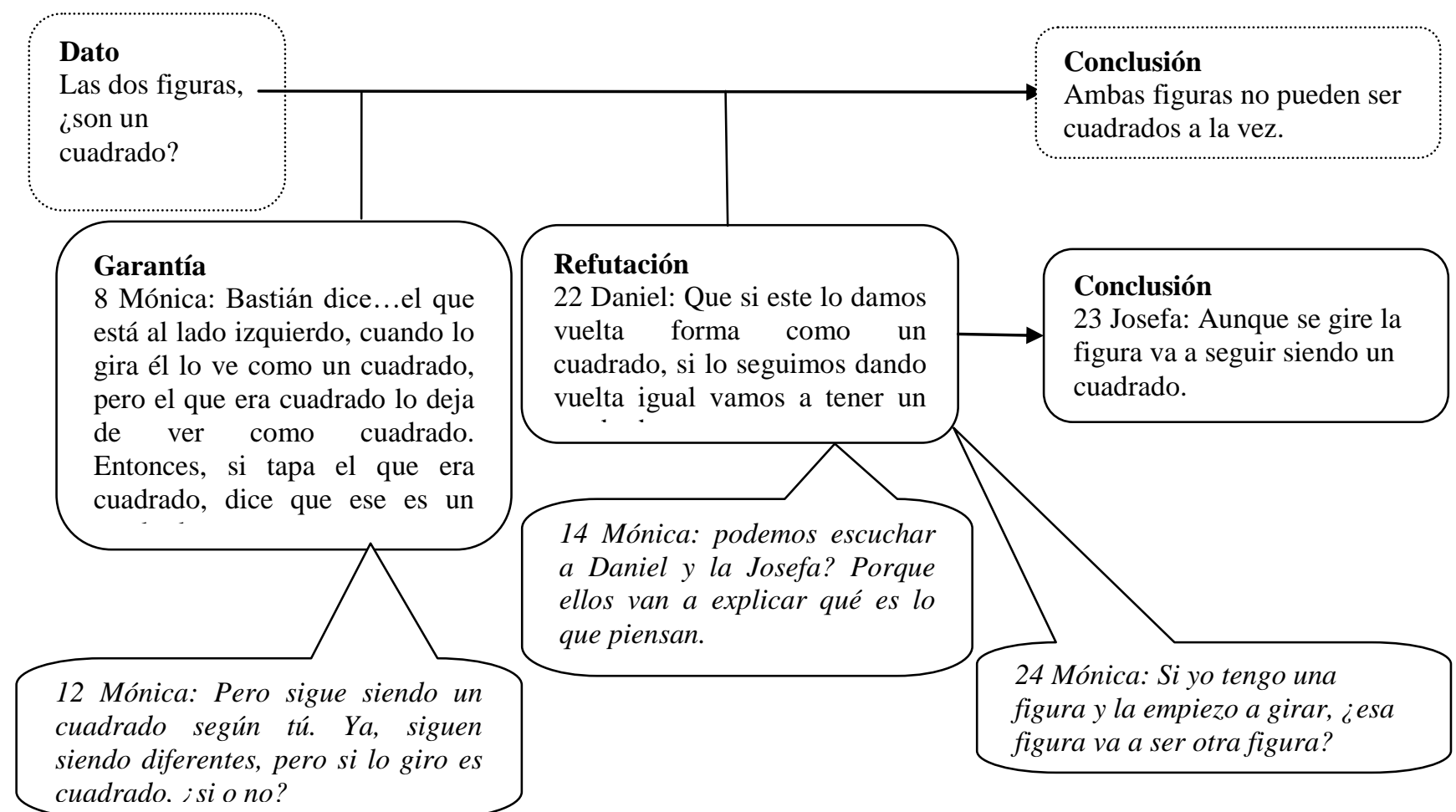

Figura 3- Mapa de la argumentación colectiva de la clase de Mónica según el modelo de Toulmin

Las intervenciones de Mónica, marcadas en los globos, han promovido que se generara la argumentación colectiva: la intervención 12 pretende elicitar ante el curso la idea de Bruno. La intervención 14 genera el espacio para que exista la contraposición de ideas; en particular la pregunta que hace Mónica (24) después de la intervención de Daniel y Josefa es un camino hacia el respaldo, Mónica hace un cambio relevante al preguntar si cualquier figura va a seguir siendo la misma si se gira en vez de centrarse solo en el cuadrado. No obstante no lo clasificamos como respaldo porque no se conecta con los movimientos rígidos - no emergió la rotación como concepto - y no es una idea desarrollada por los propios alumnos. La profesora no propone acciones para que realmente se instale el respaldo de la argumentación colectiva, a pesar de haberse dado la oportunidad para que así fuese. A manera de síntesis de estos análisis, constatamos que en las clases en las que aparece argumentación, esta está constituida por una sola estructura de los cuatro elementos mencionados.

d) Rol del docente para promover el desarrollo de la argumentación

El estudio de las estrategias comunicativas (CHAPIN; O'CONNOR; ANDERSON, 2009; LEE, 2010; SMITH; STEIN, 2011; BOERST et al., 2011), son claves, a nuestro entender, para analizar la gestión de la argumentación. Por ello, hemos diseñado un instrumento de análisis, a partir de ocho estrategias comunicativas, que se ha aplicado para el análisis de los casos seleccionados. La manera de aplicar el instrumento ha consistido en 
identificar el logro de cada indicador mediante cuatro niveles (destacado, observado, no se puede observar, se observa negativamente), registrando la cita del diálogo o comentario que permita dar evidencia del indicador. En base a la calidad y cantidad de indicadores por estrategias, se determina cuales son preponderantes en cada caso. En el Cuadro 1 se aprecia un extracto del instrumento para la estrategia comunicativa oportunidades de participación aplicado al caso de la clase de Mónica.

\begin{tabular}{|c|c|c|c|}
\hline Estrategias & Indicadores & $\begin{array}{l}\text { Nivel de } \\
\text { logro }\end{array}$ & Evidencia o comentario. \\
\hline \multirow[b]{2}{*}{$\begin{array}{l}\text { Asegurar que } \\
\text { todos tengan la } \\
\text { oportunidad de } \\
\text { aportar }\end{array}$} & $\begin{array}{l}\text { Incluir, en las } \\
\text { actividades, } \\
\text { preguntas que } \\
\text { favorezcan la } \\
\text { descripción y } \\
\text { explicación de } \\
\text { procedimientos e } \\
\text { ideas. }\end{array}$ & Destacado & $\begin{array}{l}\text { A medida que se presenta o se desarrolla la actividad, } \\
\text { la profesora realiza preguntas como: } \\
\text { - ¿Todos los lados en todas son iguales? } \\
\text { - ¿Tú estás de acuerdo con que esos dos son iguales? } \\
\text { - ¿Nos puede decir por qué usted no marcó igual que } \\
\text { la Francisca? }\end{array}$ \\
\hline & $\begin{array}{l}\text { No validar las } \\
\text { respuestas de los } \\
\text { alumnos antes de la } \\
\text { socialización de } \\
\text { algunas respuestas y } \\
\text { de las explicaciones } \\
\text { de las técnicas, ni en } \\
\text { la pizarra, ni puesto } \\
\text { por puesto. }\end{array}$ & Destacado & $\begin{array}{l}\text { La profesora no valida las respuestas que otorgan los } \\
\text { alumnos, sino que pregunta a los mismos } \\
\text { compañeros si están o no de acuerdo con ello y la } \\
\text { explicación planteada. } \\
\text { P: ¿Nos puede decir por qué usted no marcó igual } \\
\text { que la Francisca? Pero díganos a todos para que } \\
\text { escuchemos } \\
\text { Michael: Porque si nosotros giramos esto derecho, } \\
\text { quedaría igual como este [Señala un cuadrado que } \\
\text { se encuentra apoyado en un vértice y el otro que se } \\
\text { apoya en una arista] } \\
\text { P: Si ese lo giramos quedaría igual como ese ¿Puede } \\
\text { girar su guía y mirar? Si lo giran, ¿queda igual que } \\
\text { el otro o no? } \\
\text { Alumnos: Si } \\
\text { P: ¿Si o no? }\end{array}$ \\
\hline
\end{tabular}

Cuadro 1- Extracto análisis de clase de Mónica de las estrategias comunicativas

Se analizaron las clases con este instrumento, y se observó que hay tres estrategias que se van repitiendo y que están impactando en el desarrollo de la argumentación: oportunidades de participación, gestión del error y tipo de preguntas. En el Cuadro 2 se presentan los indicadores que aparecen en la clase de Matilde y Mónica para cada una de estas tres estrategias comunicativas.

\begin{tabular}{|l|l|}
\hline Matilde & Mónica \\
\hline Oportunidades de participación \\
\hline - $\begin{array}{l}\text { No validar las respuestas de los alumnos antes } \\
\text { de la socialización de algunas respuestas y de las } \\
\text { explicaciones de las técnicas, ni en la pizarra, ni } \\
\text { puesto por puesto. } \\
\text { Gestionar con flexibilidad el hecho que los } \\
\text { alumnos puedan interrumpir al profesor. }\end{array}$ & $\begin{array}{l}\text { No validar las respuestas de los alumnos antes } \\
\text { de la socialización de algunas respuestas y de las } \\
\text { explicaciones de las técnicas, ni en la pizarra, ni } \\
\text { puesto por puesto. } \\
\text { Incluir, en las actividades, preguntas que } \\
\text { favorezcan la descripción y explicación de } \\
\text { procedimientos e ideas. } \\
\text { No invalidar los errores; en su socialización de } \\
\text { los errores, retomar al niño/a que originó la } \\
\text { discusión, y pedir su opinión sobre lo planteado }\end{array}$ \\
\hline
\end{tabular}




\begin{tabular}{|c|c|}
\hline & por sus compañeros. \\
\hline \multicolumn{2}{|l|}{ Gestión del error } \\
\hline 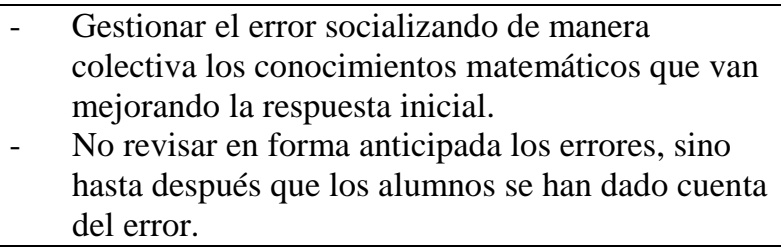 & $\begin{array}{l}\text { - Promover que alumnos con respuestas correctas e } \\
\text { incorrectas salgan a exponer, sin validar antes la } \\
\text { calidad de éstas. } \\
\text { - Gestionar el error, con foco en las explicaciones } \\
\text { incorrectas, y no en las respuestas incorrectas }\end{array}$ \\
\hline \multicolumn{2}{|l|}{ Tipo de preguntas } \\
\hline \multicolumn{2}{|c|}{$\begin{array}{ll}\text { - } & \text { Realizar preguntas que favorezcan la explicación por sobre un sí o no. } \\
\text { - } & \text { No hacer preguntas retoricas, es decir hacer la pregunta y responder inmediatamente. } \\
\text { - } & \text { Realizar contra-preguntas a los estudiantes a partir de las respuestas dadas por ellos. } \\
\text { - } & \text { Plantear preguntas que no cambien de un foco a otro muy rápidamente; tratar que las preguntas promuevan } \\
\text { que las ideas evolucionen. }\end{array}$} \\
\hline
\end{tabular}

Cuadro 2- Estrategias de participación en las clases de Matilde y Mónica

Oportunidades de participación: esta estrategia tiene la finalidad de asegurar que todos tengan la oportunidad de aportar. En el caso de Matilde el primer indicador destacado está asociado a no validar, y es un indicador que está presente en Matilde a lo largo de toda su clase, y que podemos evidenciar de manera particular en el inicio del episodio en las preguntas que hace Matilde para promover la refutación, en vez de valorar las respuesta errónea de Roberto y quedarse con la respuesta inicial que dan algunos alumnos antes de que aparezca la explicación de Roberto que hace de garantía. El segundo indicador destacado está asociado la flexibilidad que tienen los alumnos para intervenir en la discusión, el cual también se aprecia a lo largo de toda la clase, y en particular en la variedad de alumnos que intervienen. Matilde tiene la particularidad de ir relevando intervenciones clave, como la de Francisco, y da espacio a alumnos como Isabel.

En el caso de Mónica, el primer indicador destacado es: incluir actividades o preguntas que favorezcan la explicación de ideas. Se puede apreciar de la tabla 1 que Mónica hace preguntas que promueven la elicitación del pensamiento de los estudiantes. El segundo indicador destacado es: no validar las respuestas. Del Cuadro 1 se aprecia que Mónica no valida las intervenciones de los alumnos, sino que pregunta a los compañeros si están de acuerdo con ello. El tercer indicador destacado es: no invalidar los errores. Se puede observar que la profesora en ningún momento invalida los errores que aparecen sobre si el cuadrilátero girado es un cuadrado o no, sino que son los propios alumnos que argumentan que ambos cuadriláteros son un cuadrado. El hecho que se presente en ambas profesoras el indicador de no validar las respuestas de los alumnos antes de la socialización de las respuestas, no es una casualidad, ya que en los otros casos estudiados que no reportamos en este artículo, también se aprecia que es un indicador clave para las oportunidades de participación.

Gestión del error: esta estrategia tiene la finalidad de asegurar a los estudiantes que sus ideas/respuestas equivocadas son importantes para construir el conocimiento matemático. En 
la gestión de Matilde ambos indicadores destacados están muy presentes, y podemos señalar que estos indicadores son los que permiten que se genere la discusión entre las dos respuestas (12 y 24) y el proceso argumentativo descrito.

En el caso de la clase de Mónica, se destacan otros dos indicadores que se aprecian cuando se genera el contraste de ideas entre Daniel y Josefa, que salen a exponer a la pizarra sus ideas, y Bastián desde su asiento que defiende la idea errónea que al girar una de las figuras deja ser un cuadrado.

Tipo de preguntas: esta estrategia pone el foco en la formulación de preguntas adecuadas por parte del docente. Los cuatro indicadores que aparecen en el Cuadro 2 son destacados tanto en la clase de Matilde como de Mónica y se centran en preguntas que: favorecer la explicación, evitar que sean retóricas, contra-preguntas, y no cambiar el foco. Varias de las preguntas que responden a estos indicadores aparecen descritas en los globos cuando se analiza la estructura argumentativa de Toulmin. Como resultado de este análisis podemos agregar que hay un tipo de preguntas que favorecen especialmente el desarrollo de argumentación. En especial queremos destacar el indicador asociado a preguntas que no cambien de foco, ya que las interacciones, tanto de Matilde como de Mónica, que hemos asociado a este indicador han sido cruciales para generar dos puntos de vista, que es base para que exista argumentación.

Las tres estrategias comunicativas descritas son especialmente relevantes para gestionar la argumentación. En particular vemos que el tipo de preguntas es especialmente importante para la gestión especializada de la argumentación; en cambio las otras dos estrategias sirven de apoyo para promover la argumentación, ya que sin participación es difícil que aparezca argumentación, y la gestión del error promueve la contraposición de ideas.

Las estrategias comunicativas por si solas no aseguran que se promueva eficazmente la argumentación. Una de las clases observadas y que no ha sido reportada en este estudio, fue la clase de Karina, quien despliega varias estrategias comunicativas y, si bien intenta promover la argumentación, esta no se produce ya que los estudiantes presentan dificultades en la comprensión de la tarea matemática. En vista de eso nos dimos cuenta que es necesario que el docente pueda planificar la clase, cautelando que se den las condiciones para una adecuado desarrollo de la argumentación, por lo que decidimos conducir las últimas cinco sesiones del seminario a diseñar dos clases para promover la argumentación. Para ello diseñamos un plan de clases con espacios específicos para que el docente anticipe los procesos argumentativos de los estudiantes y describa el tipo de acciones y preguntas que hará para promover dichos 
procesos. En el Cuadro 3 se describen las dimensiones del diseño de una clase para promover la argumentación.

\begin{tabular}{|l|l|}
\hline \multicolumn{2}{|c|}{ Tarea matemática } \\
\hline \multicolumn{2}{|c|}{ <actividad que se realizará en la clase, se identifican las preguntas que se realizarán a los estudiantes > } \\
\hline Gestión de la Tarea matemática & $\begin{array}{l}\text { Respuestas o Procedimientos o Posturas } \\
\text { Esperadas }\end{array}$ \\
\hline $\begin{array}{l}\text { <describir los pasos e intervenciones del profesor de la tarea } \\
\text { matemática que conducen hacia el conflicto> }\end{array}$ & $\begin{array}{l}\text { <anticipar las intervenciones de los } \\
\text { estudiantes en relación a Respuestas o } \\
\text { Procedimientos o Posturas Esperadas> }\end{array}$ \\
\hline $\begin{array}{l}\text { Gestión de la confrontación de posturas } \\
\text { <hacer preguntas para gestionar el conflicto, por ejemplo que inviten a que existan más de una postura, que } \\
\text { emerjan refutaciones, e intervenciones que desarrollen el ponerse de acuerdo. Además se pueden anticipar las } \\
\text { intervenciones de los alumnos en relación a la estructura de Toulmin (dato, garantía, conclusión etc.) > }\end{array}$ \\
\hline
\end{tabular}

Cuadro 3- Diseño de una clase para promover la argumentación

Aunque la tarea matemática y su gestión son aspectos habituales en el diseño de una clase, en este modelo se pone el acento en que el docente conduce la discusión de la clase hacia el conflicto. Del mismo modo, las respuestas esperadas de los estudiantes también son esperables en el diseño de una clase, pero aquí se hace hincapié en que se expongan las posturas esperadas. Finalmente la gestión de la confrontación de posturas no es habitual en el diseño de una clase, y es la dimensión más relevante en este modelo, porque aquí se pueden anticipar las posibles estructuras argumentativas y también las intervenciones del docente que promueven la aparición de dichas estructuras.

\section{e) Condiciones para promover la argumentación en la clase de matemáticas}

En base a los puntos anteriores, en este punto podemos desarrollar la pregunta de la investigación que tiene relación con las condiciones para promover la argumentación en la clase de matemáticas. Una primera condición, expuesta en el punto anterior, corresponde a las estrategias comunicativas; un docente con dominio de ellas promueve la argumentación en clases de matemáticas. De las ocho estrategias comunicativas, las tres ya nombrabas: oportunidades de participación; gestión del error y tipo de preguntas, son las más significativas para su desarrollo. En las clases analizadas si bien aparecen diferentes indicadores relevantes en cada una de las tres estrategias, hay algunos indicadores que se repiten: en oportunidades de participación el indicador relevante que aparece es No validar las respuestas de los alumnos antes de la socialización de algunas respuestas; en gestión del error aparecen dos indicadores relevantes: Gestionar el error socializando de manera colectiva los conocimientos matemáticos y No revisar en forma anticipada los errores, sino hasta después que los alumnos se han dado cuenta del error; finalmente, en tipo de preguntas se identifican tres indicadores relevantes: Realizar actividades con preguntas que favorezcan 
la explicación por sobre un sí o no, Realizar contra-preguntas a los estudiantes a partir de las respuestas dadas por ellos y Plantear preguntas que no cambien de un foco a otro muy rápidamente. A partir de nuestros datos, podemos afirmar que la presencia de estos indicadores favorece el desarrollo de la argumentación en la clase.

Otra condición tiene relación con las características de las tareas matemáticas. Tareas abiertas que no necesariamente hay un único resultado correcto o que su desarrollo requiere de un acercamiento con estrategias informales de resolución, promueven distintos puntos de vista y con ello, el debate en los estudiantes para que exista argumentación. En cambio, tareas cerradas, especialmente las que promueven el uso de un algoritmo estándar, dificultan la aparición de la argumentación. En las últimas sesiones del seminario de formación en que se ha estado trabajando en el diseño de clases en que se promueva la argumentación, uno de los aspectos que se les ha hecho más difícil a los profesores es la elección de una tarea matemática no cerrada. En efecto, ha habido casos en que el equipo de formadores ha sugerido a una profesora una tarea abierta, pero ella la ha modificado convirtiéndola en una tarea cerrada.

Finalmente, una tercera condición tiene relación con el plan de clase. No solo basta con una tarea matemática abierta, sino que es necesario una gestión del docente que conduzca hacia el conflicto, y cuando éste se genere, el docente intervenga mediante acciones y preguntas que permitan desarrollar la argumentación. Esto lo pudimos apreciar en la clase de la profesora Mónica en que ella planificó los conflictos emergentes entre los estudiantes y gestionó la clase para que surgieran dos puntos de vista.

El plan de clases debe contemplar no solo la anticipación de las respuestas, procedimientos o posturas de los estudiantes, sino también la anticipación de sus procesos argumentativos; y en cuanto a la gestión del docente, deben diseñarse acciones y preguntas para promover dichos procesos argumentativos, por ejemplo la refutación.

En el esquema de la figura 4 se caracterizan las tres condiciones que hemos desarrollado con los respectivos indicadores. 


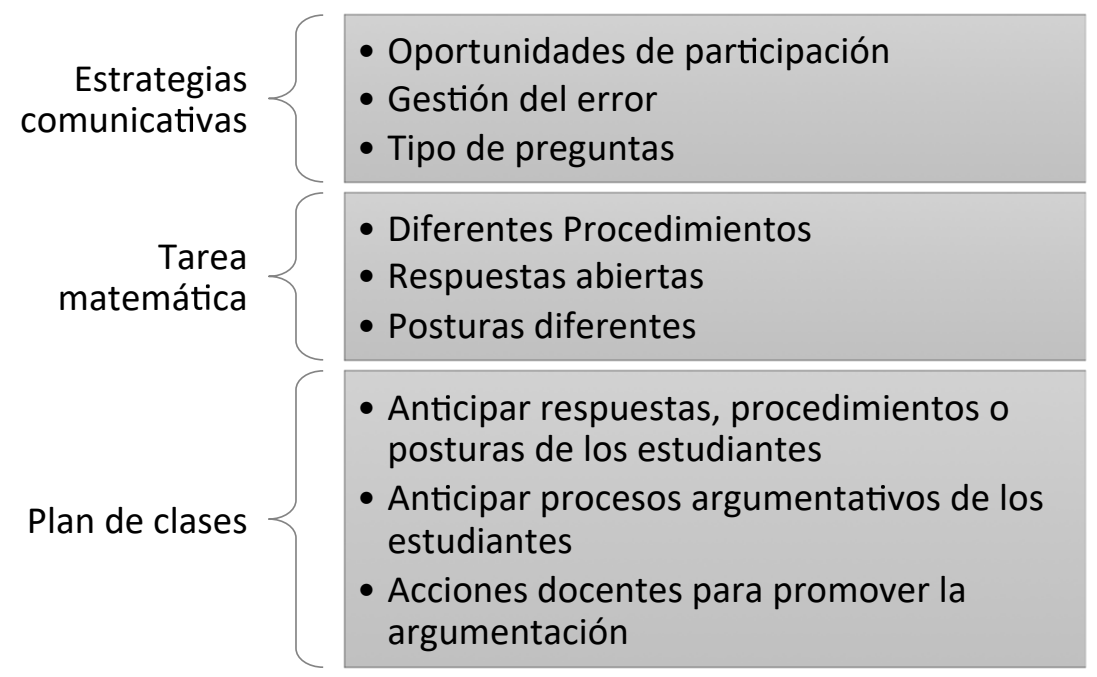

Figura 4- Condiciones para el desarrollo de la argumentación en el aula de matemáticas

\section{Conclusiones}

El desarrollo de las competencias matemáticas en la actividad escolar es un tema que está presente en gran parte de los currículum, pero al poner en práctica el desarrollo de estas competencias matemáticas en el aula, se carece de investigaciones y experiencias que ayuden al profesor a gestionarlas. En el caso de la argumentación, el profesor tiene poca familiaridad con esta competencia puesto que no ha estado presente en sus procesos de formación inicial y continua en las que, el aprendizaje de contenidos prevalecía frente al desarrollo de competencias. En consecuencia, el profesor maneja pocas herramientas para la gestión de la argumentación en el aula de matemáticas. No obstante, la importancia de desarrollar competencias en clase como la argumentación constituye una preocupación del profesorado, lo que vemos reflejado en el interés que despertó el seminario de formación en torno a la argumentación.

Al inicio del seminario, las concepciones del profesorado es que la argumentación y la explicación son coincidentes. Por medio de la Metodología de Trabajo Docente (MTD) (SOLAR et al., 2012), en que se articula el estudio de un tema a través del análisis de la práctica, los profesores se apropiaron de la idea de que la argumentación va mucho más allá de la explicación. En los casos estudiados, esta visión de argumentación se ve reflejada en sus clases, dado que existen momentos en que los estudiantes requieren refutar, y ello fue promovido por el uso de estrategias comunicativas de las profesoras. La dificultad de encontrar prácticas de argumentación en las clases de matemáticas, no depende solo del 
docente, sino que es necesario que los estudiantes estén acostumbrados a debatir sobre sus ideas.

En nuestro estudio hemos diseñado ocho estrategias comunicativas, basadas en las estrategias propuestas por Lee (2010), y a través del estudio de dos casos hemos constatado que tres de estas estrategias: oportunidades de participación, gestión del error, y tipo de preguntas, han sido las más relevantes para promover la argumentación. En particular hemos profundizado en la caracterización de estas estrategias comunicativas mediante la elaboración de indicadores para su gestión. Algo que, a nuestro entender, no está presente en la literatura sobre discusión en el aula de matemáticas (BOERST et al., 2011; CHAPIN; O'CONNOR; ANDERSON, 2009; SMITH; STEIN, 2011).

Además de las relevancias de las estrategias comunicativas, hemos evidenciado otras dos condiciones para promover la argumentación: tareas matemáticas abiertas y una planificación que anticipe los momentos potencialmente argumentativos y describa la gestión del docente en dichos momentos utilizando las estrategias comunicativas.

Finalmente, queremos destacar que un proceso de formación con profesores para promover la argumentación requiere de un tiempo considerable, y no es viable en un formato de capacitación de corto plazo, ya que para muchos profesores requiere romper barreras que solo es posible con un análisis profundo de la práctica. En este sentido, la MTD como modelo para el seminario ha resultado una plataforma adecuada para modificar de forma articulada las prácticas y concepciones de los profesores sobre el propósito de la actividad matemática escolar con sus estudiantes, encaminada más al desarrollo de competencias matemáticas.

\section{Agradecimientos}

Esta publicación se ha financiado por medio del Fondo Nacional de Desarrollo Científico y Tecnológico (FONDECYT), proyecto 11130675, Gobierno de Chile.

\section{Referencias}

BOERST, T. et al. Preparing teachers to lead mathematics discussions. Teachers College Records, New York, v. 113, n. 12, p. 2844-2877, 2011.

BRYMAN, A. Social Research Methods. 2. ed. Oxford: Oxford University Press, 2004.

CHAPIN, S. H.; O'CONNOR, C.; ANDERSON, N. C. Classroom Discussions: using Math Talk to Help Students Learn. Sausalito: Math Solutions, 2009. 
CONNER, A. M. et al. Teacher support for collective argumentation: a framework for examining how teachers support students' engagement in mathematical activities. Educational Studies in Mathematics, Berlin, v. 86, n. 3, p. 401-401, jul., 2014.

GOIZUETA, M.; PLANAS, N. Temas emergentes del análisis de interpretaciones del profesorado sobre la argumentación en clase de matemáticas. Enseñanza de las Ciencias, Barcelona, v. 31, n. 1, p. 61-78, 2013.

KRUMMHEUER, G. The ethnography of argumentation. In: COBB, P.; BAUERSFELD, H. (Ed.). The emergence of mathematical meaning: interaction in classroom cultures. Hillsdale: Lawrence Erlbaum, 1995. p. 229-269.

LEE, C. El lenguaje en el aprendizaje de las matemáticas. Madrid: Ediciones Morata, 2010.

MINEDUC. Bases curriculares. Matemática Educación Básica. Chile: Mineduc,. 2013.

MILES, M. B.; HUBERMAN, A. M. Qualitative data analysis: an expanded sourcebook. 2. ed. Thousand Oaks: Sage Publication, 1994.

NARDI, E.; BIZA, I.; ZACHARIADES, T. 'Warrant' revisited: Integrating mathematics teachers' pedagogical and epistemological considerations into Toulmin's model for argumentation. Educational Studies in Mathematics, Berlin, v. 79, n. 2, p. 157-173, feb., 2012.

OCDE. PISA 2012 Assessment and Analytical Framework: Mathematics, Reading, Science, Problem Solving and Financial Literacy. OECD Publishing, 2013.

SMITH, M. S.; STEIN, M. K. 5 Practices for Orchestrating Productive Mathematics Discussions. EEUU: NCTM, 2011.

SOLAR, H. et al. Propuesta de un Modelo de Competencia Matemática como articulador entre el currículum, la formación de profesores y el aprendizaje de los estudiantes. Educación Matemática, Ciudad de México, v. 26, n. 2, p. 33-67, ago., 2014.

SOLAR, H.; AZCÁRATE, C.; DEULOFEU, J. Competencia de argumentación en la interpretación de gráficas funcionales. Enseñanza de las Ciencias, Barcelona, v. 30, n. 3, p. 133-154, 2012.

SOLAR, H. et al. Reflexión docente y competencias matemáticas: un modelo de trabajo con docentes. Rechiem: revista chilena de educación matemática, Santiago, v. 6, n. 1, p. 257-267, 2012.

TOULMIN, S. The uses of argument. Cambridge: Cambridge University Press, 1958.

YIN, R. Case study research: design and methods. Los Ángeles: Sage, 2014. 


\section{ERRATA}

No artigo "Condiciones para promover el desarrollo de la competencia de argumentación en el aula de matemáticas " publicado no periódico Bolema, 30 (56):10921112, na página 1092 onde se lia:

"Horacio Solar Bezmalinovic" e "Jordi Deulofeu Piquet"

leia-se:

"Horacio Solar" e "Jordi Deulofeu" 